\title{
5-Regular Graphs are 3-Colorable with Positive Probability ${ }^{\star}$
}

\author{
J. Díaz ${ }^{1}$, G. Grammatikopoulos ${ }^{2,3}$, A.C. Kaporis ${ }^{2}$, L.M. Kirousis ${ }^{2,3}$, X. Pérez ${ }^{1}$, \\ and D.G. Sotiropoulos ${ }^{4}$ \\ ${ }^{1}$ Universitat Politècnica de Catalunya, Departament de Llenguatges i Sistemes \\ Informàtics, Campus Nord - Ed. Omega, 240, Jordi Girona Salgado, \\ 1-3, E-08034 Barcelona, Catalunya \\ $\{$ diaz, xperez\}@lsi.upc.edu \\ ${ }^{2}$ University of Patras, Department of Computer Engineering and Informatics, \\ GR-265 04 Patras, Greece \\ \{grammat, kaporis, kirousis\}@ceid.upatras.gr \\ ${ }^{3}$ Research Academic Computer Technology Institute, \\ P.O. Box 1122, GR-261 10 Patras, Greece \\ ${ }^{4}$ University of Patras, Department of Mathematics, GR-265 04 Patras, Greece \\ dgs@math .upatras.gr
}

Abstract. We show that uniformly random 5-regular graphs of $n$ vertices are 3-colorable with probability that is positive independently of $n$.

\section{Introduction}

The problem of finding the chromatic number of a graph has been a cornerstone in the field of discrete mathematics and theoretical computer science. Recall that the chromatic number of a graph is the minimum number of colors needed to legally color the vertices of the graph, where a coloring is legal if no two adjacent vertices share the same color. The problem is trivial for two colors but became difficult for three or more colors. Due to the difficulty of the problem, a large effort has been made in looking into structural properties of the problem, in the hope of finding more efficient procedures which apply to larger families of graphs.

An active line of research has been the characterization of classes of graphs due to their chromatic number. In particular, intense effort has been devoted to

\footnotetext{
* The 1st, 2nd, 4th and 5th authors are partially supported by Future and Emerging Technologies programme of the EU under contract 001907 "Dynamically Evolving, Large-Scale Information Systems (DELIS)". The 1st author was partially supported by the Distinció de la Generalitat de Catalunya per a la promoció de la recerca, 2002. The 3rd and 4th authors are partially supported by European Social Fund (ESF), Operational Program for Educational and Vacational Training II (EPEAEK II), and particularly Pythagoras. Part of the research of the 4th author was conducted while visiting on a sabbatical the Departament de Llenguatges i Sistemes Informàtics of the Universitat Politècnica de Catalunya.
} 
the study of the chromatic number on random structures. One active approach consists in finding the threshold $p_{k}$ of $k$-colorability for the binomial model $G_{n, p_{k}}$. An early result in this line is presented in [10, where it is shown that, for any $d \in \mathbb{N}, d>0, \exists k \in \mathbb{N}$ such that asymptotically almost surely (a.a.s.) the chromatic number of $G_{n, d / n}$ is either $k$ or $k+1$. Recall that a sequence of events $\mathcal{E}_{n}$ holds a.a.s. if $\lim _{n \rightarrow \infty} \operatorname{Pr}\left[\mathcal{E}_{n}\right]=1$. Further results on the particular threshold $p_{3}$ for 3 -colorability can be found in the introduction of [2].

A related approach is to consider the chromatic number of random $d$-regular graphs (i.e., graphs for which every vertex has degree $d$ ). For a comprehensive review on random regular graphs, the reader is referred to [14].

For $d \geq 6$, a simple counting argument shows that $d$-regular graphs are not 3-colorable, a.a.s. In fact, Molloy and Reed [12] proved that 6-regular graphs have chromatic number at least 4, a.a.s. Achlioptas and Moore 2 proved that 4-regular graphs have chromatic number 3 with uniform positive probability (w.u.p.p.), where a sequence of events $\mathcal{E}_{n}$ holds w.u.p.p. if $\liminf _{n \rightarrow \infty} \operatorname{Pr}\left[\mathcal{E}_{n}\right]>$ 0 . The proof was algorithmic in the sense that a backtracking-free algorithm based on Brelaz' heuristic was designed and shown to produce a 3-coloring w.u.p.p. Subsequently, Achlioptas and Moore [3] showed that a.a.s. the chromatic number of a $d$-regular graph $(d \geq 3)$ is $k$ or $k+1$ or $k+2$, where $k$ is the smallest integer such that $d<2 k \log k$. They also showed that if furthermore $d>(2 k-1) \log k$, then a.a.s. the chromatic number is either $k+1$ or $k+2$. This result however gives no information for the chromatic number of either 4-regular or 5-regular graphs, apart from the known fact that a.a.s. the former have chromatic number either 3 or 4 and, the latter either 3 or 4 or 5 . Shi and Wormald [13] showed that a.a.s. the chromatic number of a 4-regular graph is 3 , that a.a.s. the chromatic number of a 6 -regular graph is 4 and that a.a.s. the chromatic number of a 5 -regular graph is either 3 or 4 . They also showed that a.a.s. the chromatic number of a $d$-regular graph, for all other $d$ up to 10 , is restricted to a range of two integers.

The above results leave open the question of whether the chromatic number of a 5 -regular graph can take the value 3 w.u.p.p., or perhaps even a.a.s.

On the other hand, building on a statistical mechanics analysis of the space of truth assignments of the 3-SAT problem, which has not been shown yet to be mathematically rigorous, and Survey Propagation (SP) algorithm for 3-SAT inspired by this analysis (see e.g. 11] and the references therein), Krząkała et al. [9] provided strong evidence that 5 -regular graphs are a.a.s. 3 -colorable by a SP algorithm. They also showed that the space of assignments of three colors to the vertices (legal or not) consists of clusters of legal color assignments inside of which one can move from point to point by steps of small Hamming distance. However, to go from one cluster to another by such small steps, it is necessary to go through assignments of colors that grossly violate the requirement of legality. Moreover, the number of clusters that contain points with energy that is a local, but not global, minimum is exponentially large. As a result, local search algorithms are easily trapped into such local minima. These considerations left as the only 
plausible alternative to try to prove that 5-regular graphs are 3-colorable w.u.p.p. in an analytic way.

In this paper, we solve in the positive the question, showing that 5-regular graphs are 3-colorable w.u.p.p. The technique used is the Second Moment Method: Let $X$ be a non-negative random variable (r.v.), then

$$
\operatorname{Pr}[X>0] \geq \frac{(\mathbf{E}(X))^{2}}{\mathbf{E}\left(X^{2}\right)} .
$$

Thus, if this ratio is $\Theta(1)$, then we have that $X>0$ w.u.p.p. This well known technique (see Remark 3.1 in [8]) was used by Achiloptas and Naor [4] to solve the long standing open problem of computing the two possible values of the chromatic number of a random graph.

To apply the Second Moment Method, we work under the configuration model (see [6]) and consider the r.v. $X$ that counts a special type of colorings, which we call the stable balanced 3-colorings. In Section 2, we give exact expressions for the first and second moments of $X$, as sums of terms where each term consists of the product of polynomial (in $n$ ) and exponential terms. In Section 3 we compute the asymptotic values of those exact expressions, which turned out to be a nontrivial task. We show that $E\left(X^{2}\right)=O\left((E(X))^{2}\right)$, so that the probability in (1) is uniformly positive. Finally, the result is transferred from configurations to random 5-regular graphs.

An important remaining open question that we are working on is the extension of our result to a.a.s. It is plausible that an affirmative answer to the question can be obtained by concentration results similar to those in 3 .

\section{Exact Expressions for the First and Second Moments}

Everywhere in this paper $n$ will denote a positive integer divisible by 6 . Asymptotics are always in terms of $n$.

In the sense of the configuration model (see, e.g., 6] or [14]), let $\mathcal{C}_{n, 5}$ be the probability space of 5-regular configurations, obtained by considering a set of $n$ vertices, labelled $1, \ldots, n$, for each of these vertices a set of 5 semi-edges, labelled $1, \ldots, 5$, and a uniform random perfect matching of all $5 n$ semi-edges. Each pair of semi-edges according to the matching defines one edge of the configuration.

Definition 1. A 3-coloring of a configuration $G \in \mathcal{C}_{n, 5}$ is called stable if for every vertex $v$ and every color $i=0,1,2$, either $v$ itself or one of its neighbors are colored by $i$. Equivalently, for no single vertex $v$ can we change its color without the appearance of an edge with the same color at its endpoints. A 3coloring is called balanced if for each $i=0,1,2$, the number of vertices with color $i$ is $n / 3$.

Given a configuration $G \in \mathcal{C}_{n, 5}$, let $\mathcal{S}_{G}$ be the class of balanced stable 3-colorings of $G$. Let $X$ be the random variable that counts the number of balanced stable 3 -colorings of $\mathcal{C}_{n, 5}$. Then, the following equations can be easily shown:

$$
\left|\left\{G \mid G \in \mathcal{C}_{n, 5}\right\}\right|=\frac{(5 n) !}{2^{5 n / 2}(5 n / 2) !}, \quad \mathbf{E}(X)=\frac{\left|\left\{(G, C) \mid G \in \mathcal{C}_{n, 5}, C \in \mathcal{S}_{G}\right\}\right|}{\left|\left\{G \mid G \in \mathcal{C}_{n, 5}\right\}\right|},
$$




$$
\mathbf{E}\left(X^{2}\right)=\frac{\left|\left\{\left(G, C_{1}, C_{2}\right) \mid G \in \mathcal{C}_{n, 5}, C_{1}, C_{2} \in \mathcal{S}_{G}\right\}\right|}{\left|\left\{G \mid G \in \mathcal{C}_{n, 5}\right\}\right|} .
$$

\subsection{First Moment}

Below we assume we are given a configuration $G$ and a balanced stable 3 -coloring $C$ on $G$. The arithmetic in the indices is modulo 3 . We start by giving some useful terminology and notation:

Definition 2. A 1-spectrum $s$ is an ordered pair of non-negative integers, $s=$ $\left(s_{-1}, s_{1}\right)$, such that $s_{-1}+s_{1}=5$ and $s_{-1}, s_{1}>0$.

Notice that there are four 1-spectra. They are intended to express the distribution of the five edges stemming from a given vertex according to the color of their other endpoint. Formally, a vertex $v$ of color $i$ is said to have 1-spectrum $s=\left(s_{-1}, s_{1}\right)$, if $s_{-1}$ out of its five edges are incident on vertices of color $i-1$ and the remaining $s_{1}$ edges are incident on vertices of color $i+1$. The condition $s_{-1}, s_{1}>0$ expresses the fact that the 3 -coloring is a stable one.

For each $i=0,1,2$ and 1 -spectrum $s$, we denote by $d(i ; s)$ the scaled (with respect to $n$ ) number of vertices of $G$ which are colored by $i$ and have 1-spectrum $s$. Then, $\sum_{s} d(i ; s)=1 / 3$ and therefore $\sum_{i, s} d(i ; s)=1$.

Let $N_{1}=\left|\left\{(G, C) \mid G \in \mathcal{C}_{n, 5}, C \in \mathcal{S}_{G}\right\}\right|$. Given any two colors $i$ and $j$, observe that there are exactly $5 n / 6$ edges connecting vertices with color $i$ and $j$, respectively.

Given a fixed sequence $(d(i ; s) n)_{i, s}$ that corresponds to a balanced stable 3 coloring, let us denote by $\left(\begin{array}{c}n \\ (d(i ; s) n)_{i, s}\end{array}\right)$ the multinomial coefficient that counts the number of ways to distribute the $n$ vertices into classes of cardinality $d(i ; s) n$ for all possible values of $i$ and $s$. Let also $\left(\begin{array}{l}5 \\ s\end{array}\right)$ stand for $\left(\begin{array}{c}5 \\ s_{-1}\end{array}\right)=\left(\begin{array}{c}5 \\ s_{1}\end{array}\right)$.

By a counting argument, we have that

$$
N_{1}=\sum_{d(i ; s)_{i, s}}\left(\begin{array}{c}
n \\
(d(i ; s) n)_{i, s}
\end{array}\right)\left(\prod_{i, s}\left(\begin{array}{l}
5 \\
s
\end{array}\right)^{d(i ; s) n}\right)\left(\frac{5 n}{6} !\right)^{3},
$$

where the summation above is over all possible sequences $(d(i ; s))_{i, s}$ that correspond to balanced stable 3-colorings.

\subsection{Second Moment}

Below we assume we are given a configuration $G$ and two balanced stable 3colorings $C_{1}$ and $C_{2}$ on $G$. For $i, j=0,1,2$, let $V_{i}^{j}$ be the set of vertices colored with $i$ and $j$ with respect to colorings $C_{1}$ and $C_{2}$, respectively. Let $n_{i}^{j}=\left|V_{i}^{j}\right| / n$, and let $E_{i}^{j}$ be the set of semi-edges whose starting vertex is in $V_{i}^{j}$. Also, for $r, t \in\{-1,1\}$, let $E_{i, r}^{j, t}$ be the set of semi-edges in $E_{i}^{j}$ which are matched with one in $E_{i+r}^{j+t}$. Let $m_{i, r}^{j, t}=\left|E_{i, r}^{j, t}\right| / n$. We have that $\sum_{r, t} m_{i, r}^{j, t}=5 n_{i}^{j}, \sum_{i, j} n_{i}^{j}=1$, and therefore $\sum_{i, j, r, t} m_{i, r}^{j, t}=5$. And, since matching sets of semi-edges should have equal cardinalities, we also have that $m_{i, r}^{j, t}=m_{i+r,-r}^{j+t,-t}$. 
Definition 3. A 2-spectrum $s$ is an ordered quadruple of non-negative integers, $s=\left(s_{-1}^{-1}, s_{-1}^{1}, s_{1}^{-1}, s_{1}^{1}\right)$, such that $s_{-1}^{-1}+s_{-1}^{1}+s_{1}^{-1}+s_{1}^{1}=5$ and $\left(s_{-1}^{-1}+s_{-1}^{1}\right)\left(s_{1}^{-1}+\right.$ $\left.s_{1}^{1}\right)\left(s_{-1}^{-1}+s_{1}^{-1}\right)\left(s_{-1}^{1}+s_{1}^{1}\right)>0$.

Notice that the number of 2 -spectra is 36 . Let $v$ be a vertex in $V_{i}^{j}$. Vertex $v$ is said to have 2-spectrum $s=\left(s_{-1}^{-1}, s_{-1}^{1}, s_{1}^{-1}, s_{1}^{1}\right)$ if $s_{r}^{t}$ out of its five edges, $r, t \in\{-1,1\}$, are incident on vertices in $V_{i+r}^{j+t}$. The condition $\left(s_{-1}^{-1}+s_{-1}^{1}\right)\left(s_{1}^{-1}+\right.$ $\left.s_{1}^{1}\right)\left(s_{-1}^{-1}+s_{1}^{-1}\right)\left(s_{-1}^{1}+s_{1}^{1}\right)>0$ expresses the fact that both $C_{1}$ and $C_{2}$ are stable.

For each $i, j=0,1,2$ and 2 -spectrum $s$, we denote by $d(i, j ; s)$ the scaled number of vertices which belong to $V_{i}^{j}$ and have 2 -spectrum $s$. We have:

$$
\sum_{s} s_{r}^{t} d(i, j ; s)=m_{i, r}^{j, t}, \quad \sum_{s} d(i, j ; s)=n_{i}^{j} \quad \text { and therefore } \sum_{i, j, s} d(i, j ; s)=1 .
$$

Throughout this paper we refer to the set of the nine numbers $n_{i}^{j}$ as the set of the overlap matrix variables. We also refer to the set of the thirty-six numbers $m_{i, r}^{j, t}$ as the set of the matching variables. Finally, we refer to the $9 \times 36$ numbers $d(i, j ; s)$ as the spectral variables.

Let $N_{2}=\mid\left\{\left(G, C_{1}, C_{2}\right)\left|G \in \mathcal{C}_{n, 5}, C_{1}, C_{2} \in \mathcal{S}_{G}\right|\right.$. Given a fixed sequence $(d(i, j ; s) n)_{i, j, s}$ that corresponds to a pair of balanced stable 3 -colorings, let us denote by $\left(\begin{array}{c}n \\ (d(i, j ; s) n)_{i, j, s}\end{array}\right)$ the multinomial coefficient that counts the number of ways to distribute the $n$ vertices into classes of cardinality $d(i, j ; s) n$ for all possible values of $i, j$ and $s$. Let also $\left(\begin{array}{l}5 \\ s\end{array}\right)$ stand for $\left({ }_{s_{-1}^{-1}, s_{-1}^{1}}^{5}, s_{1}^{-1}, s_{1}^{1}\right)$ (the distinction from a similar notation for 1-spectra will be obvious from the context). Now, by an easy counting argument, we have:

$$
N_{2}=\sum_{d(i, j ; s)_{i, j, s}}\left\{\left(\begin{array}{c}
n \\
(d(i, j ; s) n)_{i, j, s}
\end{array}\right)\left(\prod_{i, j, s}\left(\begin{array}{l}
5 \\
s
\end{array}\right)^{d(i, j ; s) n}\right)\left(\prod_{i, j, r, t}\left(\left(m_{i, r}^{j, t} n\right) !\right)^{\frac{1}{2}}\right)\right\}
$$

where the summation above is over all possible sequences $(d(i, j ; s))_{i, j, s}$ that correspond to pairs of balanced stable 3-colorings.

\section{$3 \quad$ Asymptotics}

In this section we will show that $E\left(X^{2}\right)=O\left((E(X))^{2}\right)$. An immediate consequence of this is that 5-regular configurations have a balanced stable 3 -coloring and hence a generic 3-coloring w.u.p.p.

By applying Stirling approximation to formulae (2), (4) and (5), we get:

$$
\begin{gathered}
\mathbf{E}(X) \sim \sum_{d(i ; s)_{i, s}} f_{1}\left(n, d(i ; s)_{i, s}\right)\left(6^{-\frac{5}{2}} \prod_{i, s}\left(\frac{\left(\begin{array}{c}
5 \\
s
\end{array}\right)}{d(i ; s)}\right)^{d(i ; s)}\right)^{n} \\
\mathbf{E}\left(X^{2}\right) \sim \sum_{d(i, j ; s)_{i, j, s}} f_{2}\left(n, d(i, j ; s)_{i, j, s}\right)\left[5^{-\frac{5}{2}} \prod_{i, j, s}\left(\frac{\left(\begin{array}{l}
5 \\
s
\end{array}\right)}{d(i, j ; s)}\right)^{d(i, j ; s)}\left(\prod_{i, j, r, t}\left(m_{i, r}^{j, t}\right)^{\frac{1}{2} m_{i, r}^{j, t}}\right)\right]^{n}
\end{gathered}
$$


where $f_{1}$ and $f_{2}$ are functions that are sub-exponential in $n$ and also depend on the sequences $d(i ; s)_{i, s}$ and $d(i, j ; s)_{i, j, s}$, respectively. By a result similar to Lemma 3 in [3], by using a standard Laplace-type integration technique, we can prove that the Moment Ratio is asymptotically positive if

$$
(E(X))^{2} \asymp E\left(X^{2}\right) \text {, i.e. } \ln \left((E(X))^{2}\right) \sim \ln \left(E\left(X^{2}\right)\right) .
$$

Let $M_{1}$ be the maximum base $6^{-5 / 2} \prod_{i, s}\left(\frac{\left(\begin{array}{c}5 \\ s\end{array}\right)}{d(i ; s)}\right)^{d(i ; s)}$ as $d(i ; s)_{i, s}$ ranges over all possible sequences that correspond to balanced stable 3-colorings. Analogously, let $M_{2}$ be the maximum base

$$
5^{-5 / 2}\left(\prod_{i, j, s}\left(\frac{\left(\begin{array}{l}
5 \\
s
\end{array}\right)}{d(i, j ; s)}\right)^{d(i, j ; s)}\right)\left(\prod_{i, j, r, t}\left(m_{i, r}^{j, t}\right)^{\frac{1}{2} m_{i, r}^{j, t}}\right) .
$$

From the equations (6) and (7) one can immediately deduce that the relation (8) is true if $\left(M_{1}\right)^{2}=M_{2}$. We need to compute the exact values of $M_{1}$ and $M_{2}$.

\subsection{First Moment: Computing $M_{1}$}

Let $f=\prod_{i, s}\left(\frac{\left(\begin{array}{c}5 \\ s\end{array}\right)}{d(i ; s)}\right)^{d(i ; s)}$ be a real function of 12 non-negative real variables $d(i ; s)$ defined over the polytope $\sum_{s} d(i ; s)=1 / 3$, where $i=0,1,2$ and $s$ runs over 1-spectra. The following lemma follows from the application of elementary analysis techniques and the computation of Lagrange multipliers.

Lemma 1. The function $\ln f$ is strictly convex. Let $\mathcal{D}_{1}=\sum_{i, s}\left(\begin{array}{l}5 \\ s\end{array}\right)=3 \times 30$. The function $f$ has a maximizer at the point where $d(i ; s)=\frac{\left(\begin{array}{l}5 \\ s\end{array}\right)}{\mathcal{D}_{1}}, \forall i, s$.

By direct substitution, we obtain:

Lemma 2. $M_{1}=6^{-5 / 2}\left(\prod_{i, s} \mathcal{D}_{1}^{d(i, s)}\right)=\left(\frac{1}{6}\right)^{5 / 2} \mathcal{D}_{1}=\sqrt{\frac{25}{24}}$.

From the above and from (6), we get:

Theorem 1. The expected number of balanced stable 3-colorings of a random 5 -regular configuration approaches infinity as $n$ grows large.

\subsection{Second Moment: Computing $M_{2}$}

$$
\text { Let } \quad F=\left(\prod_{i, j, s}\left(\frac{\left(\begin{array}{l}
5 \\
s
\end{array}\right)}{d(i, j ; s)}\right)^{d(i, j ; s)}\right)\left(\prod_{i, j, r, t}\left(m_{i, r}^{j, t}\right)^{\frac{1}{2} m_{i, r}^{j, t}}\right)
$$

be a real function of non-negative real variables $d(i, j ; s)$ (where $i, j=0,1,2$, $r, t=-1,1$ and $s$ runs over 2 -spectra) defined over the polytope determined by:

$$
\sum_{j, s} d(i, j ; s)=1 / 3, \forall i ; \sum_{i, s} d(i, j ; s)=1 / 3, \forall j \text { and } m_{i, r}^{j, t}=m_{i+r,-r}^{j+t,-t},
$$

where $m_{i, r}^{j, t}=\sum_{s} s_{r}^{t} d(i, j ; s)$. Notice that $F$ is a function of $9 \times 36$ variables. 
We will maximize $F$ in three phases. In the first one, we will maximize $F$ assuming the matching variables $m_{i, r}^{j, t}$ are fixed constants such that their values are compatible with the polytope over which $F$ is defined. Thus we will get a function $F_{m}$ of the 36 matching variables $m_{i, r}^{j, t}$. At the second phase we will maximize $F_{m}$ assuming that the nine overlap matrix variables $5 n_{i}^{j}=\sum_{r, t} m_{i, r}^{j, t}$ are fixed constants compatible with the domain of the matching variables. Thus we will get a function $F_{n}$ of the overlap matrix variables. The preceding two maximizations will be done in an analytically exact way. Observe that since we consider balanced 3-colorings, $F_{n}$ depends only on the values of four n's. We will maximize $F_{n}$ by going through its 4-dimensional domain over a fine grid. Let us point out that the maximizations above will not be done ex nihilo. Actually, we know (see below) the point where we would like the maximizer to occur. Therefore all we do is not find the maximizer but rather prove that it is where we want it to be. The proof of the next lemma is done by direct substitution.

Lemma 3. Let $\mathcal{D}_{2}=\sum_{i, j, s}\left(\begin{array}{l}5 \\ s\end{array}\right)=9 \times 900$ and let $d(i, j ; s)=\left(\begin{array}{l}5 \\ s\end{array}\right) / \mathcal{D}_{2}, \forall i, j, s$. Then the value of the base $5^{-5 / 2} F$ at the above values of $d(i, j ; s)_{i, j, s}$ is equal to $\left(M_{1}\right)^{2}=25 / 24$.

We call the sequence $d(i, j ; s)=\left(\begin{array}{l}5 \\ s\end{array}\right) / \mathcal{D}_{2}$ the barycenter. Barycenter as well we call the corresponding point in the domain of $F_{m}$, i.e. the point $m_{i, r}^{j, t}=5 / 36$, $\forall i, j, r, t$. Finally, barycenter also we call the corresponding point in the domain of $F_{n}$, i.e. the point $n_{i}^{j}=1 / 9, \forall i, j$. We will see, by direct substitutions, that the functions $5^{-5 / 2} F_{m}$ and $5^{-5 / 2} F_{n}$ as well take the value $\left(M_{1}\right)^{2}=25 / 24$ at their corresponding barycenters. Therefore, after computing $F_{m}$ and $F_{n}$, all that will remain to be proved is that $F_{n}$ has a maximizer at its barycenter $n_{i}^{j}=1 / 9$, $i, j=0,1,2$. Below, we compute the functions $F_{m}$ and $F_{n}$ and then we show that the barycenter is a maximizer for $F_{n}$ by sweeping its 4 -dimensional domain.

From the Spectral to the Matching Variables. Everywhere below we assume that the 36 matching variables $m_{i, r}^{j, t}$ are non-negative and moreover take only values for which there exist $9 \times 36$ spectral non-negative variables $d(i, j, s)$ such that

$$
m_{i, r}^{j, t}=\sum_{s} s_{r}^{t} d(i, j ; s), i, j=0,1,2, r, t=-1,+1 .
$$

and such that the equations in (10) hold. It is not hard to see that the above restrictions on the matching variables are equivalent to assuming that $\forall i, j=$ $0,1,2$ and $\forall r, t=-1,1$,

$$
\begin{gathered}
m_{i, r}^{j, t}=m_{i+r,-r}^{j+t,-t}, \quad \sum_{i, r, t} m_{i, r}^{j, t}=5 / 3, \quad \sum_{j, r, t} m_{i, r}^{j, t}=5 / 3 \text { and } \\
m_{i, r}^{j, t} \geq 0, \quad m_{i, r}^{j, t}+m_{i, r}^{j,-t} \leq 4\left(m_{i,-r}^{j, t}+m_{i,-r}^{j,-t}\right), \quad m_{i, r}^{j, t}+m_{i,-r}^{j, t} \leq 4\left(m_{i, r}^{j,-t}+m_{i,-r}^{j,-t}\right) .
\end{gathered}
$$

Fix such values for the $m_{i, r}^{j, t}$. To maximize the function $F$ given by equation (9) over the polytope described in (10) for the fixed values of the matching variables $m_{i, r}^{j, t}, i, j=0,1,2, r, t=\{-1,1\}$, it is sufficient to maximize the function $F$ subject to the 36 constraints in (11). We call this maximum $F_{m}$. 
Since for different pairs of $(i, j), i, j=0,1,2$, neither the variables $d(i, j ; s)$ nor the constraints in (11) have anything in common, and since the matching variables are fixed, it is necessary and sufficient to maximize separately for each $i, j=0,1,2$ the function $F_{i, j}=\prod_{s}\left(\left(\begin{array}{l}5 \\ s\end{array}\right) / d(i, j ; s)\right)^{d(i, j ; s)}$, subject to the four constraints: $\sum_{s} s_{r}^{t} d(i, j ; s)=m_{i, r}^{j, t}, r, t=-1,1$. We will use Lagrange multipliers to maximize the logarithm of these functions. Notice that the functions $\ln F_{i, j}$ are strictly convex. We define the following function:

$\Phi(x, y, z, w)=(x+y+z+w)^{5}-(x+y)^{5}-(x+z)^{5}-(y+w)^{5}-(z+w)^{5}+x^{5}+y^{5}+z^{5}+w^{5}$.

Also for each of the nine possible pairs $(i, j), i, j=0,1,2$, consider the $4 \times 4$ system:

$$
\frac{\partial \Phi\left(\mu_{i,-1}^{j,-1}, \mu_{i,-1}^{j, 1}, \mu_{i, 1}^{j,-1}, \mu_{i, 1}^{j, 1}\right)}{\partial \mu_{i, r}^{j, t}} \quad \mu_{i, r}^{j, t}=m_{i, r}^{j, t}, \quad r, t=-1,1,
$$

where $\mu_{i, r}^{j, t}$ denote the 36 unknowns of these nine $4 \times 4$ systems. Applying the method of the Lagrange multipliers, we get

Lemma 4. Each of the nine systems in (14) has a unique solution. Moreover in terms of the solutions of these systems

$$
F_{m}=\prod_{i, j, r, t}\left(\frac{\left(m_{i, r}^{j, t}\right)^{\frac{1}{2}}}{\mu_{i, r}^{j, t}}\right)^{m_{i, r}^{j, t}}
$$

By the above Lemma, we have computed in an analytically exact way the function $F_{m}$. Notice that the function $F_{m}$ is a function of the 36 matching variables $m_{i, r}^{j, t}, i, j=0,1,2$ and $r, t=-1,1$, over the domain given by (12) and (13). However its value is given through the solutions of the systems in (14), which have a unique solution.

From the Matching to the Overlap Matrix Variables. We assume now that we fix nine non-negative overlap matrix variables $n_{i}^{j}$ such that $\sum_{i} n_{i}^{j}=1 / 3$, $\forall j$ and $\sum_{j} n_{i}^{j}=1 / 3, \forall i$. Using again multiple Lagrange multipliers, we will find the maximum, call it $F_{n}$, of the function $F_{m}$ given in Lemma 4 under the constraints:

$$
\sum_{r, t} m_{i, r}^{j, t}=5 n_{i}^{j}, \quad \text { and } m_{i, r}^{j, t}=m_{i+r,-r}^{j+t,-t}, \text { for } i, j=0,1,2 \text { and } r, t=\{-1,1\} .
$$

assuming in addition that the $m_{i, r}^{j, t}$ satisfy the inequalities in (13). We consider the latter inequality restrictions not as constraints to be dealt with Lagrange multipliers, but as restrictions of the domain of $F_{m}$ that must be satisfied by the maximizer to be found.

We will need that the function $\ln F_{m}$ over the polytope determined by the constraints (15) (for fixed values of the variables $n_{i}^{j}$ ) is strictly convex. To show this it is sufficient to fix an arbitrary $i, j=0,1,2$ and show that the 4 -variable function $\ln \left(\prod_{r, t}\left(\left(m_{i, r}^{j, t}\right)^{\frac{1}{2}} / \mu_{i, r}^{j, t}\right)^{m_{i, r}^{j, t}}\right)$, subject to the single linear constraint 
$\sum_{r, t} m_{i, r}^{j, t}=n_{i}^{j}$, is strictly convex. To show the latter, we computed the Hessian and its LPMD's after solving the single linear constraint for one of its variables (thus we obtained a function of three variables). Notice that the value of the function under examination is given through the unique solutions of a $4 \times 4$ system. The Hessian and the LPMD's were analytically computed in terms of these solutions by implicit differentiation of the equations of the system. The strict convexity then would follow if we showed that at every point of the domain of this 3-variable function, the LPMD's were non-zero and of alternating sign. We demonstrated this by going over this domain over a fine grid and computing at all its points the LPMD's. The values of the LPMD's that we got were safely away from zero and with the desired algebraic sign. Notice that although to prove the convexity of the function $\ln F_{m}$, subject to the constraints in (15), we relaxed the constraints $m_{i, r}^{j, t}=m_{i+r,-r}^{j+t,-t}$, the latter ones are essential for correctly computing $F_{n}$.

To apply Lagrange multipliers, we have to find the partial derivatives of the function $\ln F_{m}=\sum_{i, j, r, t}\left(\frac{1}{2} m_{i, r}^{j, t} \ln m_{i, r}^{j, t}-m_{i, r}^{j, t} \ln \mu_{i, r}^{j, t}\right)$. In fact, after a few manipulations, we obtain:

Lemma 5. $\sum_{r^{\prime}, t^{\prime}} m_{i, r^{\prime}}^{j, t^{\prime}} \frac{\partial \ln \mu_{i, r^{\prime}}^{j, t^{\prime}}}{\partial m_{i, r}^{j, t}}=\frac{1}{5}$, and thus $\frac{\partial \ln F_{m}}{\partial m_{i, r}^{j, t}}=\frac{3}{10}+\frac{1}{2} \ln m_{i, r}^{j, t}-\ln \mu_{i, r}^{j, t}$

By applying now the technique of multiple Lagrange multipliers, we get:

Lemma 6. Consider the $45 \times 45$ system with unknowns $\mu_{i, r}^{j, t}$ and $x_{i}^{j}$ :

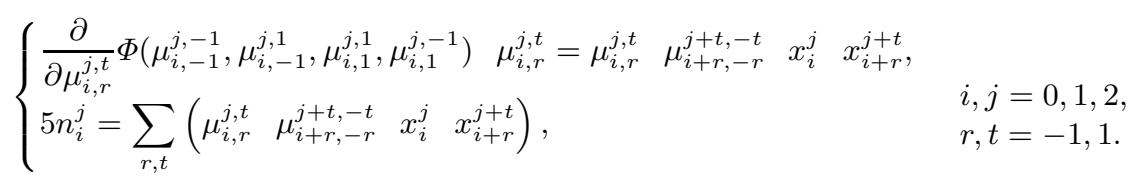

This system has a unique solution. Moreover in terms of the solution of this system:

$$
F_{n}=\prod_{i, j}\left(x_{i}^{j}\right)^{5 n_{i}^{j}}
$$

So we have computed in an analytically exact way the function $F_{n}$. Since solving the $45 \times 45$ system in Lemma 6 when $n_{i}^{j}=1 / 9, i, j=0,1,2$ is trivial, we get by direct substitution:

Lemma 7. The value of $5^{-5 / 2} F_{n}$ at the barycenter $n_{i, j}=1 / 9, i, j=0,1,2$ is equal to $\left(M_{1}\right)^{2}=25 / 24$. Therefore the value of $F_{n}$ at the barycenter $i s>58.2309$.

Therefore all that it remains to be proved is that the function $F_{n}$ maximizes at the barycenter.

From the Overlap Matrix Variables to the Conclusion. We have to prove the function $F_{n}$ maximizes at the barycenter. Since we have assumed that the 3coloring is balanced, i.e. $\forall i, \sum_{j} n_{i}^{j}=1 / 3$ and $\forall j, \sum_{i} n_{i}^{j}=1 / 3$, the domain of $F_{n}$ has four degrees of freedom, all in the range $[0,1 / 3]$. We swept over this domain 
going over the points of a grid with 200 steps per dimension. The sweeping avoided a thin layer (of width 1/1000) around the boundary (the points in the domain where at least one of the $n_{i}^{j}=0$ ), because at the boundary the derivative of the original function $F$ is infinity, thus no maximum occurs there. Moreover, we have computed the Hessian at the barycenter and proved that it is negative definite so in an neighborhood of the barycenter $F_{n}$ is convex, and we know that $F_{n}$ will be smaller than at the barycenter. At all points where we got a value for $F_{n}$ greater than 58, we made an additional sweep at their neighborhood of step-size 1/7500 (all these points where close the barycenter). Nowhere did we get a value greater than the value at the barycenter. To solve the $45 \times 45$ systems efficiently, we designed a fast search algorithm based on an algorithm by Byrd et al. [7. We also devised a way to select good starting points for each system, whose basic principle was to select for each successive system a starting point that belonged to the convex hull of the solutions to the previous systems. The algorithm was implemented in Fortran and run on the IBM's supercomputer in the Barcelona Supercomputing Center, which consists of 2.268 dual 64-bit processor blade nodes with a total of $4.5362 .2 \mathrm{GHz}$ PPC970FX processors. Therefore,

Theorem 2. Random 5-regular configurations are 3-colorable with uniformly positive probability.

In order to transfer this result to random 5-regular graphs, we need to consider the restriction of $\mathcal{C}_{n, 5}$ to simple configurations (i.e. those without loops and multiple edges). We write $\mathbf{P r}^{*}$ and $\mathbf{E}^{*}$ to denote probability and expectation conditional to the event " $G \in \mathcal{C}_{n, 5}$ is simple". By using similar techniques to the ones developed in [5] (see also Theorem 2.6 in [14]), we get:

Lemma 8. Let $C$ be any fixed balanced 3 -coloring of $n$ vertices. Then, $\operatorname{Pr}[G$ is simple $\mid C$ is stable coloring of $G]$ is bounded away from 0 , independently of $C$ and $n$.

From this lemma, we obtain: $\mathbf{E}^{*}(X)=\Theta(\mathbf{E}(X))$ and $\mathbf{E}^{*}\left(X^{2}\right)=O\left(\mathbf{E}\left(X^{2}\right)\right)$. Therefore, $\mathbf{P r}^{*}[X>0] \geq \frac{\left(\mathbf{E}^{*}(X)\right)^{2}}{\mathbf{E}^{*}\left(X^{2}\right)}=\Theta(1)$, and we can conclude:

Theorem 3. The chromatic number of random 5-regular graphs is 3 with uniformly positive probability.

\section{Acknowledgement}

We wish to thank D. Achlioptas and C. Moore for their essential help at all phases of this research, without which we would not have obtained the results of this work. We are also indebted to N.C. Wormald for his useful suggestions and we are thankful to the Barcelona Supercomputing Center and in particular to David Vicente for the help in running the optimization programs on the Mare Nostrum supercomputer. 


\section{References}

1. D. Achlioptas and C. Moore. The asymptotic order of the random $k$-SAT threshold. In: Proc. 43th Annual Symp. on Foundations of Computer Science (FOCS), 126$127,2002$.

2. D. Achlioptas and C. Moore. Almost all graphs with degree 4 are 3-colorable. Journal of Computer and Systems Sciences 67(2), 441-471, 2003.

3. D. Achlioptas and C. Moore. The chromatic number of random regular graphs. In: Proc. 7th International Workshop on Approximation Algorithms for Combinatorial Optimization Problems (APPROX) and 8th International Workshop on Randomization and Computation (RANDOM) (Springer, LNCS, 2004) 219-228.

4. D. Achlioptas and A. Naor. The two possible values of the chromatic number of a random graph. In: 36th Symposium on the Theory of Computing (STOC), 587-593, 2004 .

5. B. Bollobás. A probabilistic proof of an asymptotic formula for the number of labelled regular graphs. European Journal of Combinatorics 1, 311-316, 1980.

6. B. Bollobás. Random Graphs. Academic Press, London-New York, 1985.

7. R.H. Byrd, P. Lu and J. Nocedal. A limited memory algorithm for bound constrained optimization. SIAM Journal on Scientific and Statistical Computing 16(5), 1190-1208, 1995.

8. S. Janson, T. Łuczak and A. Ruciński. Random Graphs. John Wiley, 2000.

9. F. Krzạkała, A. Pagnani and M. Weigt. Threshold values, stability analysis and high-q asymptotics for the coloring problem on random graphs. Phys. Rev. E 70, 046705 (2004).

10. T. Łuczak. The chromatic number of random graphs Combinatorica 11, 45-54, 1991.

11. M. Mézard and R. Zecchina. Random K-satisfiability: from an analytic solution to a new efficient algorithm. Phys.Rev. E 66, 056126 (2002).

12. M. Molloy. The Chromatic Number of Sparse Random Graphs. Master's Thesis, University of Waterloo, 1992.

13. L. Shi and N. Wormald. Colouring random regular graphs Research Report CORR 2004-24, Faculty of Mathematics, University of Waterloo, 2004.

14. N.C. Wormald. Models of random regular graphs. In: J.D. Lamb and D.A. Preece, eds., Surveys in Combinatorics (London Mathematical Society Lecture Notes Series, vol. 267, Cambridge U. Press, 1999) 239-298. 